\title{
FACTORS AFFECTING RECOVERY OF SENSORY FUNCTION AFTER NERVE LESIONS
}

\author{
BY
}

\author{
E. GUTMANN and L. GUTTMANN
}

From the Department of Zoology and Comparative Anatomy, Oxford

\author{
(Received 7TH August, 1942)
}

Most of our knowledge of the processes of sensory recovery after nerve lesions is derived from human cases. Indeed, in some ways man is most suitable for such work, because he can give verbal information about the quality of sensation which is felt. But there are many problems which can only be solved by the comparison of recovery under conditions which differ in respect of one variable only, say level of lesion. Human cases are rarely sufficiently comparable to allow this to be done. Complications are introduced by such factors as varying length of nerve that has been crushed, the time between injury and operation, skill of the surgeon, and so on.

It is somewhat surprising, therefore, that there has been remarkably little detailed study of sensory recovery in animals. Vanlair (1882 and 1887) studied recovery of sensation on the sole of dogs after various operative procedures, and several workers have used sensory recovery among other signs for testing the suitability of surgical procedures (Takimoto 1917, Huber 1919, Gosset and Bertrand 1938, Tangari 1940, Muesch and Roessel 1941). Most of the observations on recovery of sensation following the interruption of a nerve in animals are restricted to a brief statement that recovery of sensation had taken place after a certain time. Such statements are both insufficient and unsatisfactory. It will be seen later that recovery of sensation in a denervated area is a complex process and only a part of it is due to regeneration of the interrupted nerve. An exact description of the denervated area in which recovery is observed is thus necessary before any conclusions may be drawn. Therefore a clear knowledge of the sensory distribution of various nerves is an essential preliminary to the study of recovery of sensation in animals. In rabbits there is no satisfactory account of the areas innervated by the various peripheral nerves. No doubt the reason for this lack of investigation has been the fear that reflex responses of the animal would not be sufficiently consistent to enable accurate mapping of the sensory state of the skin. However, if properly handled, rabbits may serve as satisfactory " observers." The responses which may be most conveniently used in the rabbit are withdrawal reactions to pin prick stimuli.

The work reported here may be said to have three objects: first, to delimit the maximal and autonomous areas of the skin served by various nerves of the lower extremities of the rabbit; secondly, to show the manner in which shrinkage of the insensitive area left after denervation takes place, and the parts played in this shrinkage by $(a)$ recovery in the zone of overlap between the interrupted nerve and the neighbouring nerves; $(b)$ recovery in the autonomous zone of the interrupted nerve; and thirdly, to show how recovery is affected by such factors as the nature of the lesion, its distance from the skin, age of the animal, and infection.

\section{Methods}

The rabbits used fall into two series: (1) 60 in which the nerves were cut but reunion was prevented by resection of several centimetres; (2) 94 in which the nerves were interrupted, either (a) by complete crushing at a single point with watchmakers' forceps (69 cases), or (b) by severing with scissors and suturing with concentrated plasma (22 cases) (Young and Medawar, $1940)$, or (c) crushing the nerve over a length of $4 \mathrm{~cm}$. ( 3 cases). Series 1 was further subdivided into those animals in which the nerve whose sensory distribution was being studied was resected in order to delimit its autonomous zone, and other animals in which the nerve under study was left intact but all the nerves supplying adjacent skin areas were resected in order to delimit the maximal zone of the nerve in question.

All operations were performed aseptically under nembutal and ether anesthesia. At various times after operation the area of cutaneous analgesia was tested by pin prick. The needle was inserted vertically through the thickness of the skin, always working from the zone of sensory loss towards the sensitive zone. The points at which the first withdrawal reactions occurred were marked with indian ink or 30 per cent. silver nitrate. These single points were then joined by lines, which thus roughly mark the area of analgesia. Photographs were made as often as possible, or alternatively the areas were recorded on outline drawings of the rabbit's foot. For this latter purpose it was convenient to take measurements from certain points of the foot, namely, the external and internal malleolus or the line between them, the malleolar line, the base of the 5th metacarpus, the line of the tendon of the M. tibialis anterior where it crosses the malleolar line, and the crista tibix.

Before giving a detailed description of the results obtained in the individual nerves some general points may be mentioned which have to be considered in sensory tests on rabbits. The reaction of a rabbit to cutaneous nociceptive stimuli consists in an increase of the respiration rate, increased movement of the nares, and in local or general 
withdrawal reaction. The intensity of reaction is not determined solely by the intensity of the stimulus. Age, race, and general condition of the animal have a definite influence on the kind and intensity of reaction. Younger animals generally react more briskly than adults, but the responses are sometimes more inconstant. Of the different races albinos are found relatively less reliable "witnesses" to pin stimuli. Yet even within a single race hyperreactive and hyporeactive types may be distinguished. General bad condition, caused by infection, sores, etc., undoubtedly raise the threshold of sensitivity. These variations have to be remembered when interpreting results such as are shown in the figures on the following pages. They also make it undesirable to repeat examinations at too frequent intervals: in nearly all our cases the tests have been done once a week. A further important point is that the various parts of the body have not the same threshold to nociceptive stimuli. In the posterior limb the sole and the areas below the outer and inner ankles are normally more sensitive than the dorsum of the foot or the anterior and medial parts of the leg. In the forelimbs the palmar aspect of the paw showed a lower threshold to pin-prick than the dorsum.

\section{Peroneal Nerve}

After isolated division of the peroneal nerve in the thigh the area of cutaneous analgesia at first extends over a large portion of the anterior surface of the shank as well as over the dorsum of the foot (Fig. 1). During the immediately succeeding days

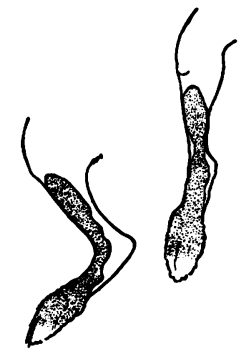

A

B

Fig. 1.-Outline of the area of sensory loss after isolated section of the peroneal nerve, $(a)$ lateral view, $(b)$ anterior view. The shaded area is analgesic.

the area shrinks considerably, especially on the shank. The time and rate of this shrinkage shows clearly that it cannot be due to return of peroneal fibres to the area. The recovery of pain sensibility is therefore due to fibres passing in other nerves, particularly the lateral cutaneous nerve of the thigh which supplies the upper third of the shank. Indeed, the sensory loss in this area of the shank is very variable and depends to a certain extent on the amount of operative damage to other nerves. Thus, after high lesions of the peroneal, which involve an extensive opening in the thigh, the area of analgesia is always larger than after lesions at the level of the knee. In the first case the direct or indirect damage to the lateral cutaneous nerve of the thigh is presumably more extensive than in the second. When the operation is done quickly and carefully there may be a shorter period at which response to pin prick is entirely absent on the front of the shank. On the other hand, after manipulation of the neighbouring nerves the loss is especially profound and recovery is delayed.

It is not clear why " recovery" in such an intermediate area should not take place immediately after operation, except of course in cases of damage to the neighbouring nerves. It might be expected that the threshold of pain fibres running from the part of the skin of the front of the leg which is supplied by adjacent nerves would be unaffected by severance of the axons of the peroneal nerve, even though the two sets mingle in the cutaneous plexus. There is, however, some such disturbance, since the area of analgesia is greater in the days immediately after operation than later. In fact, the "recovery" of the pain fibres of the adjacent nerves proceeds progressively, occurring first in the upper part of the front of the leg, and last just above the malleolar line, i.e. in the area closest to the autonomous zone of the peroneal, where there are presumably the fewest intact fibres. It may be noted, however, that in a number of cases the whole intermediate zone, even after complete recovery, remained hypoalgesic. There is not sufficient information available to decide the nature of this " recovery" or adjustment of function of the undamaged over lapping fibres in an intermediate zone. We do not know to what degree it occurs centrally or peri pherally but it will be seen later that the peripheral factor is very important.

Early shrinkage of the analgesia on the foot is less marked than on the front of the leg, and the area of overlap of the peroneal nerve with the sural on the lateral and the saphenous major on the medial borders is far less extensive than with the lateral cutaneous nerve of the thigh. Some 14 to 30 days after the lesion, in young animals even within a few days, when recovery in the intermediate zone is complete, there remains only analgesia of the autonomous zone of the peroneal. This extends over most of the dorsum of the foot, from the line between the malleoli downwards, but excludes small triangular areas in the inner and outer ankle regions which are supplied by the saphenous and sural nerves.

The participation of the peroneal nerve in the cutaneous supply of the rabbit's leg may also be shown by determining the maximal zone supplied by this nerve. After section of all adjacent nerves, i.e. tibial, sural, cutaneous femoris lateralis and saphenous major, the area of remaining sensibility has been found to include not only the whole dorsum of the foot but also the anterior, antero-lateral and antero-medial aspects of the leg, up to a level $38 \mathrm{~mm}$. above the malleolar line. The response to pin-prick in the whole of this area is as brisk as in the corresponding areas of the normal leg. One day after operation some responses, though inconstant, have 
been found as high as $58 \mathrm{~mm}$. above the malleolar line.

Returning to the phenomena which follow a crushing of the peroneal nerve. After the early rapid recovery of pain sensibility on the front of the leg, there follows a period during which the margin of algesia remain about at the malleolar line. When a large section has been removed from the nerve the margin remains at this level indefinitely. There is very little penetration of fibres at this point from neighbouring nerves into the autonomous zone of the peroneal by local extension (Weddell et al., 1941). However, if the peroneal nerve has been simply crushed, or the ends sutured after cutting, this period of waiting ends after a time which varies with the position and nature of the lesion. Indeed, it is not easy to determine the exact end. Once responses have begun to appear to pin prick in the autonomous zone there follows a more or less regular advance down the foot until the whole becomes sensitive. The advance is faster and more regular after a crush than it is after severance and suture. Figs. $2(a)$ and $(b)$ give good examples

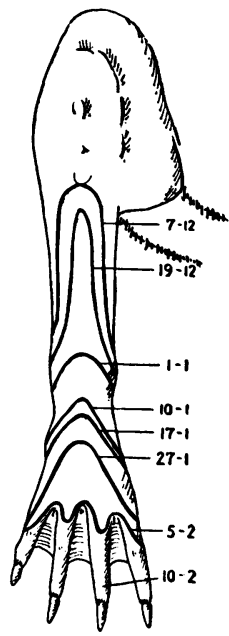

A

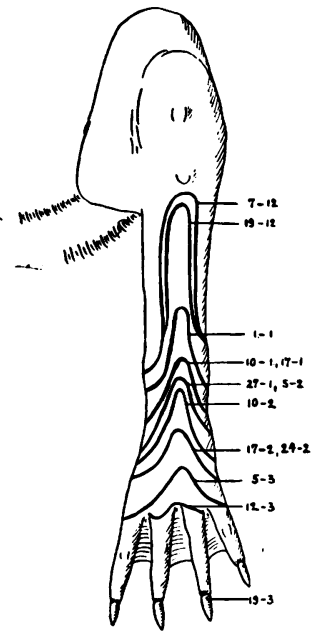

B
FIG. 2.-Diagrams showing the progress of recovery on the two sides of one animal (a) after crushing, (b) after suturing the peroneal nerve in middle of thigh. The operations were performed on 5.12.41, and complete recovery was recorded on 10.2.41 after the crush and on 19.3.41 after the suture. The pointers indicate the dates at which the various examinations were made.

of the two conditions. It is seen that recovery is slower after suturing even in the intermediate zone. This may be explained by the more extensive and longer manipulations which are necessary for suturing a nerve. After the crush each weekly examination showed some advance of the margin, whereas after suture there appeared to be periods of stagnation.

It has, however, been noticed that in all cases recovery proceeds from above downwards along the foot. The recovery is usually a little further advanced at the edges of the foot than at the centre of the area, which has the effect of producing the $\mathrm{V}$-shaped lines shown. It is therefore not necessary to postulate that recovery takes place in any more complex manner than by simple growth of new fibres from the old trunks down into the plexuses.

In the distal parts of the autonomous zone of the peroneal nerve recovery of sensation proceeds somewhat quicker on the dorsum of the toes than in the digital interspatia, thus forming a waveshaped line (Fig. 3).

During the study of recovery after lesions of the

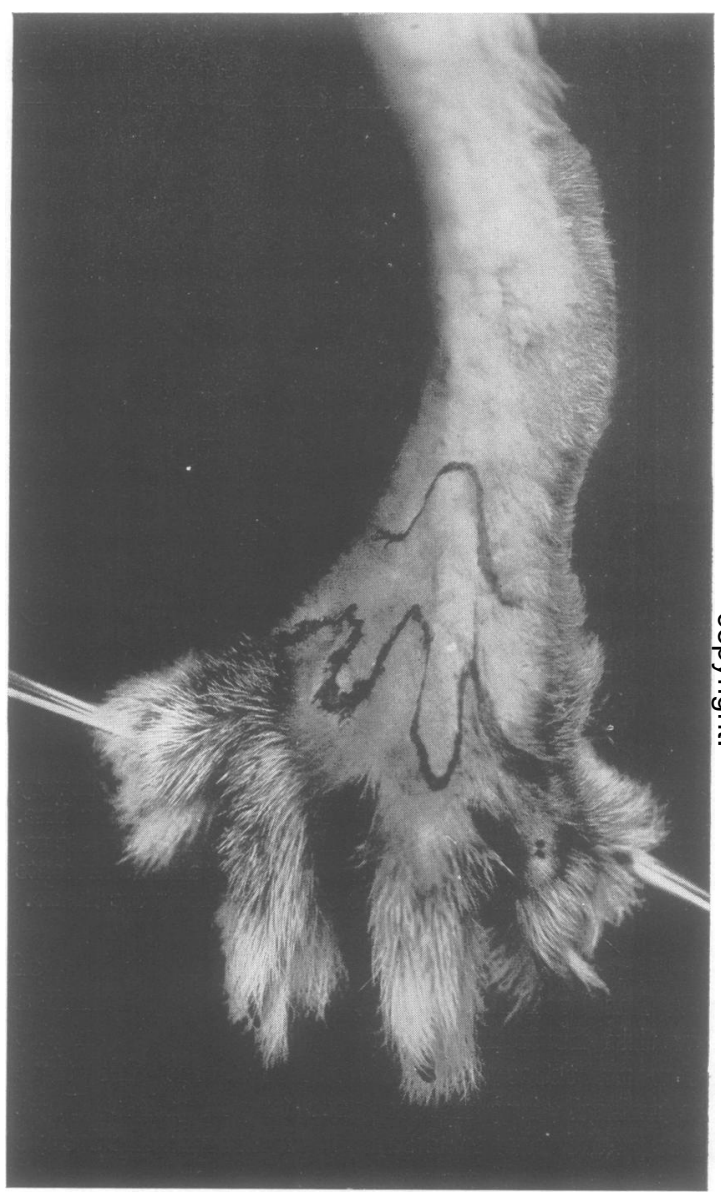

FIG. 3.-Sensory recovery in the autonomous zone of the peroneal nerve after crushing in the middle of the thigh. The V-shaped line on the middle of the dorsum of the foot represents the border line of the analgesic area 53 days after operation, the wavy line represents the border line of analgesia 7 days later.

peroneal nerve the question arises, whether or not there is a period between arrival of fibres and their functional recovery. This question of a " peripheral delay" could be approached by calculations of rate of regeneration and by histological examinations. From the figures of Gutmann et al. (1942) we may calculate that after crushing the nerve at the malleolar line the first new fibres must have arrived back in the skin 7 days after the operation. Actually rezovery in the autonomous zone start 
as late as 12 days after operation (see Fig. 4). The extra period until recovery begins must be occupied either by slower growth of fibres within the skin, or in waiting for the arrival of a sufficient number of fibres. Since the pain fibres are presumably unmedullated there can hardly be any long period of waiting for maturation within the nerve trunk. Some indication of the time and nature of the " peripheral delay" may be found by histological examination. If a crush is performed high up in the thigh fibres should arrive in the main trunk on the dorsum of the foot about 60 days after operation (Gutmann et al. 1942). Histological observations showed that a piece of skin removed from the middorsum of the foot at this time does not contain

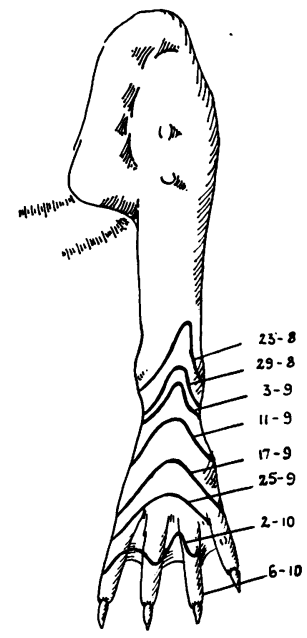

FIG. 4.-Diagram showing the progress of recovery after crushing the peroneal nerve in the malleolar line. The operation was performed on the 21.8.41, complete recovery was recorded on the 6.10 .41 . The pointers indicate the dates at which the various examinations were made.

any fibres. But in an animal in which a piece of skin has been removed 69 days after operation a few thin fibres may be seen, though most of the Schwann tubes contain no fibres. At this time the animal gives no response to pin prick. In a piece of skin from the same region removed 95 days after operation there are more fibres in the skin and they appear thicker, but many Schwann tubes still contain no fibres. No response to pin prick is obtainable at this time. The first positive response to pin prick has been obtained in one case 112 days after such an operation. The skin removed at this time shows a further increase in number of fibres. In this case it can thus be calculated that there is a period of about 40 days when, although fibres are present in the skin, no positive response is obtained. It is difficult to estimate the exact length of this delay as measurements of the length of the fibres in the skin can only be approximate and onset of recovery cannot be determined with exactness. However, it is clear that the delay is greater with the more distant lesions.

The histological findings indicate that the time between the arrival of the first fibres and their response to nociceptive stimuli is occupied by addition of new fibres and presumably a process of maturation. A certain number of fibres seems to be necessary before a positive response to nociceptive stimuli is obtainable. Thus one animal in which the peroneal nerve was sutured showed no recovery in the autonomous zone even 200 days after the operation although motor function had recovered at the usual time. Histological examination of a piece of skin removed from the middorsum of the foot showed that fibres were present in the skin but they were few and mostly thin.

Throughout this account we have not considered the possibility that some part of the sensory recovery in the denervated area is due to local extension of fibres into the denervated area from adjacent nerves (Weddell et al., 1941). This factor may well play a part in the shrinkage of the autonomous area of the peroneal. The fact that in our cases, even in the absence of re-innervation through the peroneal nerve itself, the area of analgesia remains almost constant in the autonomous zone, shows that this factor operates only to a minor extent. However, in two animals in which no recovery due to regeneration was possible considerable shrinkage of the area of analgesia within the usual autonomous zone of the peroneal was found 6 months after operation, the line of analgesia running 9 and $12 \mathrm{~mm}$. below the malleolar line. This recovery could be explained by extension of fibres derived from adjacent nerves.

\section{Tibial Nerve}

As this nerve is most frequently used in experiments on rabbits an accurate knowledge of its sensory function is of particular importance. Immediately after isolated section of the tibial nerve in the thigh an area of sensory loss is found situated over the medial two-thirds of the calcaneum, the medial half or two-thirds of the proximal and middle parts of the sole and the whole distal part of the sole except a narrow strip on the lateral border. There is also a small area of sensory loss on the medial border of the foot, encroaching on the inner ankle region and involving the proximal part of the 1st metatarsal. In the period following operation, however, a marked shrinkage of the area of sensory loss has been found in all cases, showing that there is an extensive overlap of the adjacent nerves, particularly the sural. A more or less rapid shrinkage of the zone of sensory loss is particularly obvious in the mixed zone between tibial and sural nerves, i.e. the lateral aspect of the heel and sole, forming a curved encroachment towards the medial aspect of the sole at the level of the 5th metatarsus (Fig. 5). On the other hand the border between the distribution of tibial and saphenous major remains constant for a longer time. Thus a bridge of sensory loss is formed between the sural and saphenous major distribution on the medial part of the proximal border of the sole and the foot. This bridge becomes gradually æsthetic, particularly in young animals, by the recovery of the zone of overlap with the sural nerve and by a time in which 
no recovery by calculation due to regeneration could have taken place.

The extensive overlap, especially with the sural nerve, is also shown by determining the maximal zone of the tibial nerve. After cutting all nerves supplying adjacent skin areas (sural, peroneal, and saphenous major) a considerable extension of the maximal zone takes place in the days after the operation with the result that almost the whole sole becomes sensitive (see Fig. 6). The great practical

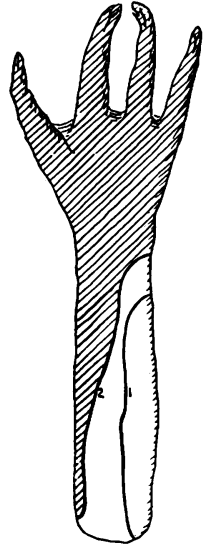

Fig. 5.-Outline of the areas of sensory loss on the plantar surface of the foot 1 and 17 days after isolated section of the tibial nerve. Line 1 represents the border of the analgesic area 1 day after operation, line 2, 17 days after operation. The shaded area represents the zone remaining analgesic 17 days after operation.

importance of this extensive overlap between tibial and sural particularly in the heel region is obvious. Saving the sural nerve at operation in experiments on the tibial portion of the sciatic nerve either prevents completely or at least greatly delays and diminishes the development of a sore at the heel region, a development which only too often frustrates long lasting experiments in rabbits.

Sensory recovery in tibial lesions due to true regeneration may only be assumed when it proceeds in the distal third of the sole and along the medial side distally from a line corresponding with the 5 th metatarsus. Recovery due to regeneration in the autonomous zone of the tibial advances in a centrifugal direction, as described in the process of recovery of the peroneal (Fig. 7). Again the margin of advance is not straight since recovery is a little more advanced at the sides than at the centre of the area.

\section{Sural Nerve}

The boot-shaped area of sensory loss following severance of this nerve and its rapid shrinkage have been described elsewhere (Weddell, Guttmann, and Gutmann, 1941). Shrinkage of the area of sensory loss after isolated section of the sural nerve is so extensive in young animals that the whole area of analgesia may recover within a period of a month. The details of the changes in the extent of the "autonomous" and maximal zone of the sural measured from the calcaneum to the distal boundaries of sensory change were measured in a 4 weeks old rabbit (No. 1). It is to be noted that the recovery of sensory function in the areas of overlap takes

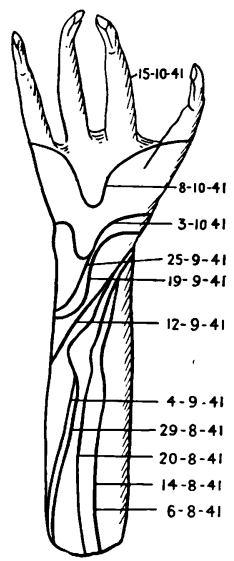

FIG. 7.-Diagram showing the progress of recovery after crushing the tibial in the lower third of the thigh. The operation was performed on 30.7 .41 , complete recovery recorded on 15.10 .41 . The pointers indicate the dates at which the different examinations were made.

place not only in the distal part but progresses from the upper and also from the other parts of the denervated sural area. Data from a further animal (No. 2) illustrate the shrinkage of the " autonomous" zone only, measured from the calcaneum to both the distal and proximal boundaries of sensory change.*

RabBit No. 1.

Time of test after operation (days)

1
8

15

26
31

Extent of " autonomous" zone

(mm.)

21

16

15

7

$\mathbf{0}$

Extent of maximal zone

(mm.)

30

34

39

41

41

41

RabBit No. 2.

Time of test after operation (days)

Extent of "autonomous" zone

$\begin{array}{cc}\begin{array}{c}\text { distal } \\ \text { boundary }\end{array} & \begin{array}{c}\text { proximal } \\ \text { boundary }\end{array} \\ 30 & 33 \\ 30 & 33 \\ 20 & 17 \\ 18 & 15 \\ 14 & 13 \\ 10 & 10 \\ 0 & 0\end{array}$

The area in which sensation was restored, particularly the centre of the sural's cutaneous distribution below the outer ankle, was found hypo-

* The fact that sensibility returns rapidly throughout the analgesic area after severance of pure skin ne rves particularly in young animals makes the term "autonomous zone" of doubtful significance (Weddell, et al., 1941). 
algesic compared with the analogous part of the opposite side. During the process of shrinkage after isolated section of the sural nerve the insensitive sural area above the outer ankle can be divided into two areas separated from each other by encroachments of overlapping nerves from either side.

The quick sensory recovery due to action of adjacent nerves makes it difficult to study the process of recovery due to regeneration of the interrupted nerve in young animals. An interesting feature during the course of recovery is a bridge formation

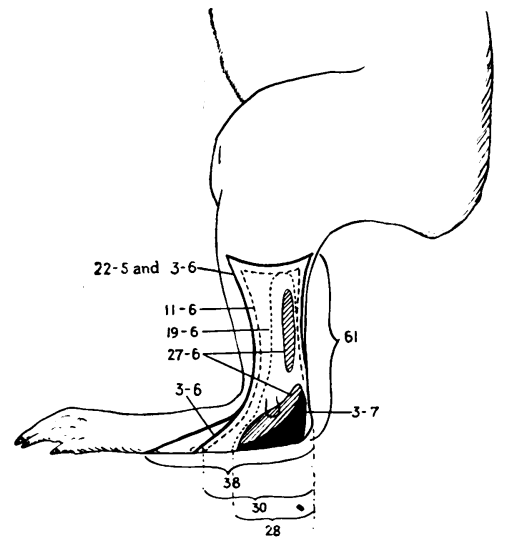

FIG. 8.-Diagram showing the progress of recovery after crushing the sural nerve in the lower third of the thigh. The operation was performed on 21.5.41, complete recovery recorded on 11.7.41. The brackets indicate the dimensions in $\mathrm{mm}$. of the areas of sensory loss during the progress of recovery. The two cross-hatched areas and the black area are the areas of sensory loss found on 27.6.41, the black area alone represents the area of sensory loss found on 3.7.41. The pointers indicate the dates at which the different examinations were made.

in the lower third of the shank which separates two analgesic fields (Fig. 8), and is similar to that described after isolated section.

\section{Postero-lateral Cutaneous Nerve of the Thigh}

After isolated section of this nerve an area of analgesia was found occupying the greater part of the lateral aspect of the thigh and tapering to the upper third of the leg (Fig. 9, first line). The length

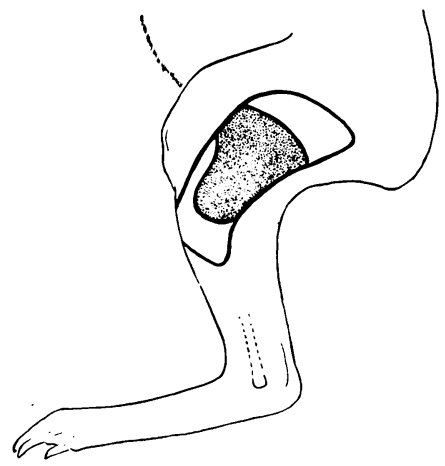

Fig. 9.-Outline of the areas of sensory loss 1 and 14 days after isolated section of the postero-latero-cutaneous nerve of the thigh. The shaded area is the area found analgesic 14 days after operation. of the whole insensitive area found in this case 3 days after operation was $98 \mathrm{~mm}$., its greatest width in the thigh was $37 \mathrm{~mm}$. and on the leg $28 \mathrm{~mm}$. In the period following operation a considerable shrinkage of both the proximal and distal borders of the area of sensory loss was found. Moreover the shrinkage is also obvious from the lateral border, particularly from the area of the antero-lateral cutaneous nerve of the thigh. Within a period of 14 days after operation the length of the insensitive zone has diminished to $59 \mathrm{~mm}$. (Fig. 9, second line). The difference between the lines in the proximal part is $17 \mathrm{~mm}$., that in the distal part $22 \mathrm{~mm}$.

\section{Saphenous Major}

Fig. 10 shows the area of sensory loss after isolated

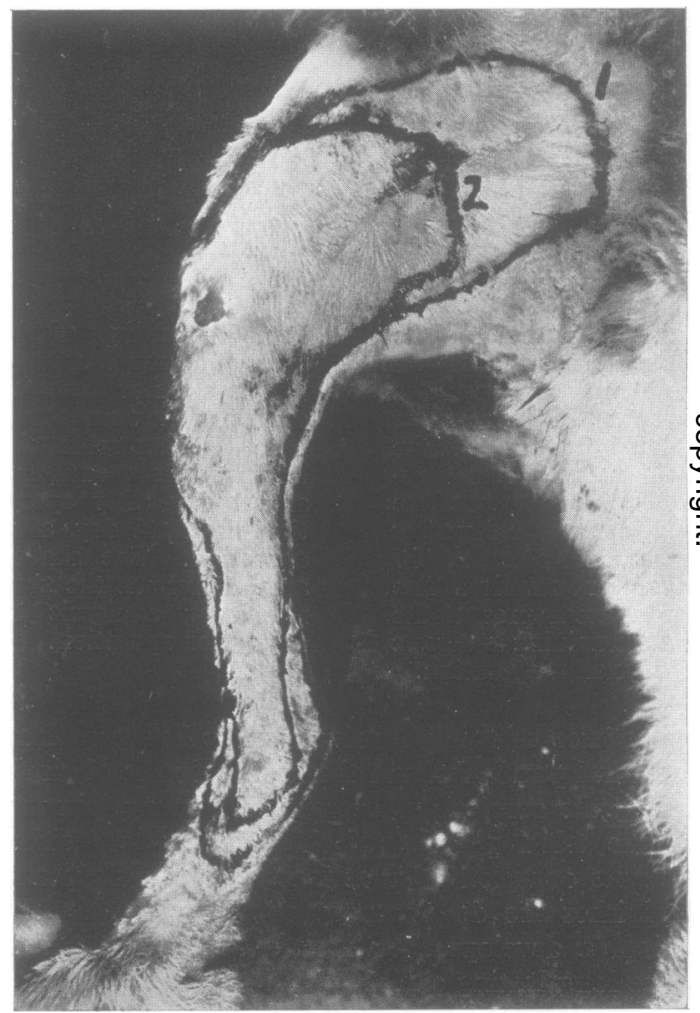

FIG. 10.-Outlines of the area of sensory loss 1 and 17 days after isolated section of the saphenous major nerve. Line 1 represents the border of the area of sensory loss 1 day after operation, line 2 the area of sensory loss 17 days after operation.

section of this nerve and the shrinkage at the proximal and distal borders 1 week after the operation. Fig. $11(a)$ and $(b)$ show that the anteromedial aspect of the shank receives fibres from both, the saphenous major and the peroneal nerves, a fact which is of interest in connection with the recovery in the area of overlap after peroneal lesion.

Fig. 12 demonstrates the sensory recovery after crushing the saphenous major in the groin. The zone of sensory loss found 1 day after operation 


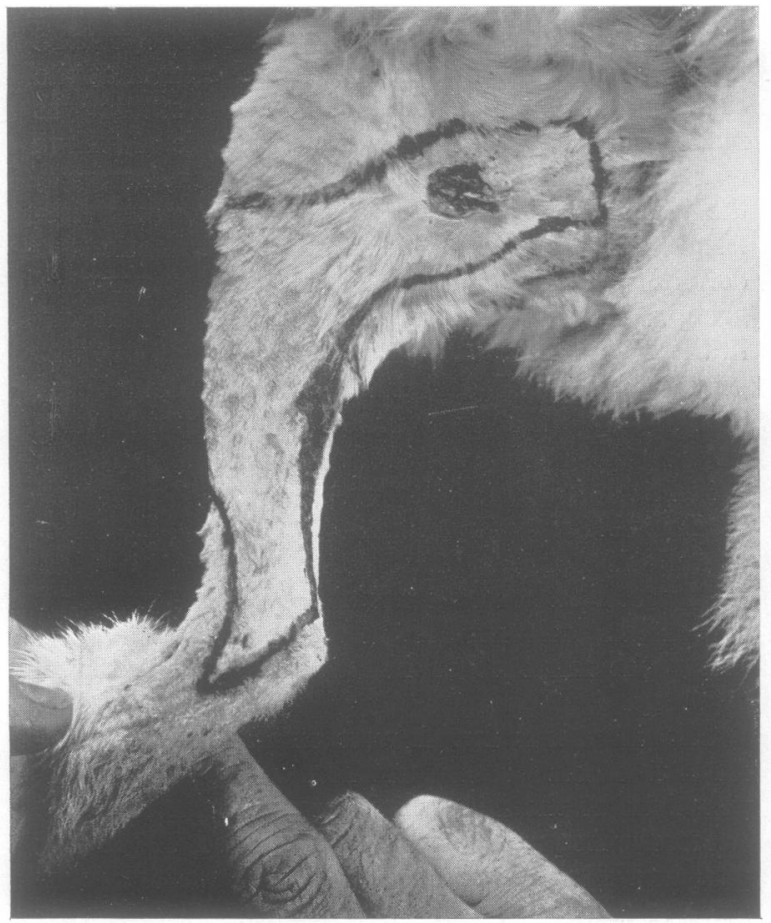

A

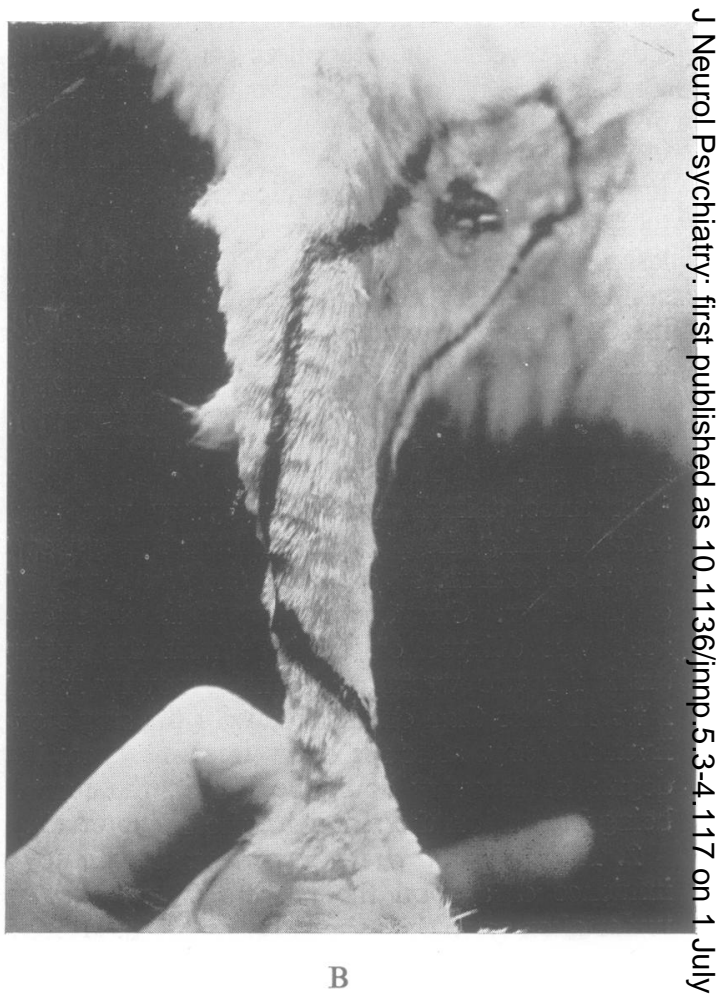

B

Fig. 11.-Outline of the area of sensory loss immediately after isolated section of the saphenous major, demonstrat $\overrightarrow{0} \vec{\varphi}$ ing the participation of the nerve in the innervation of the anteromedial aspect of the shank: $(a)$ medial viewo $\mathrm{N}$ (b) anteromedial view.

is very extensive. The shrinkage in the area of overlap begins very early after operation and the degree of shrinkage in the proximal as well as in the distal part of the area of sensory loss around the inner ankle is very remarkable in the first period after

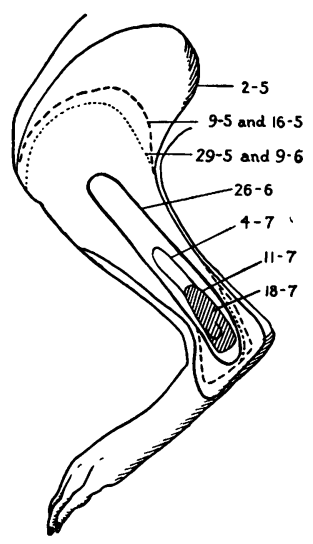

Fig. 12.-Diagram showing the progress of recovery after crushing the saphenous major in the groin. The operation was performed on 1.5 .41 , complete recovery recorded on 18.7.41. The shaded area is the area of sensory loss on 11.7.41. The pointers indicate the dates at which the various examinations were made.

operation. In the later stages of recovery, as described in other nerves, the shrinkage of the analgesic area progresses centrifugally and from either side. The case described shows a regular advance of sensory recovery. The centre of the autonomous zone of the saphenous major, localized around the inner ankle region recovers last.

\section{The effect of various factors on speed and degree of recovery}

1. Type of lesion.-A comparison between the course of recovery in a lesion in continuity on one side and division and suture of the peroneal on the other side is shown in Figs. $2(a)$ and $(b)$. In this experiment the peroneal nerve on the right side was crushed in the middle of the thigh with a fine watchmaker's forceps, and on the left side was cut at the same level and immediately sutured with plasma. Marked differences in time and degree (i.e. number and constancy of positive responses to pin prick, and the intensity of the reaction) of final recovery were found in favour of the lesion in continuity. Complete sensory recovery of the whole autonomous zone occurred after 67 days, whereas on the side of the severed and sutured nerve complete recovery took 104 days. The actual speed of advance of the line of analgesia within the autonomous zone on the sutured side was not much different from that on the crushed side, the main difference being that the advance began much later on the sutured side (see below p. 125). Even more obvious than the difference in time is the difference in degree of recovery. From the recovering autonomous zone of the crushed side the positive responses to nociceptive stimuli were 
definitely more brisk and lively than on the sutured side. Moreover the responses on the sutured side were found to be more inconstant and sometimes deep pin prick elicited only a slow and sluggish withdrawal reaction. A further difference was found in the degree of trophic changes, particularly in the development of sores. Although peroneal lesions generally do not develop sores so easily as combined tibial and sural lesions they were found in some cases on certain places, i.e. dorsum of the toes and base of the 5th metatarsal. These trophic changes were found to be larger, and recovered more slowly, after division of the peroneal, than after crush.

In three animals sensory recovery was studied after crushing the nerve with large forceps over a length of $40 \mathrm{~mm}$. on one side, and at a single point on the other. Onset of recovery in the autonomous zone was delayed on the side with the extensive crush for two weeks, and final recovery recorded 2 weeks later than on the side with the single crush. Even 200 days after the operation differences in the degree of recovery could be found, the responses being brisker on the side with the single crush.

2. Recovery and age.-Experiments to study the influence of age on the process of recovery were undertaken on rabbits about 1 month old. Recovery of sensation was followed after interruption of the peroneal, sural and tibial nerves. Eleven animals were operated on at different levels. In 3 animals with lesions of the peroneal nerve, after an initial shrinkage of the analgesic field below the malleolar line no further shrinkage could be observed although normal motor recovery occurred. No special reason for this stagnation in the process of recovery could be found.

The process of recovery shows the same general principles as described in adult animals. Shrinkage by recovery of the area of overlap in young animals proceeds very quickly. In 2 cases after peroneal lesions in the middle of the thigh the whole front of the shank has been found æsthetic even 3 days after operation. After crushing the peroneal nerve in the middle of the thigh recovery of the analgesic area was complete after about 1 month, compared with the average of 79 days in adult animals where the peroneal nerve has been crushed at this level.

After crushing the sural nerve in baby rabbits in the lower third of the thigh complete recovery was recorded in 1 month, compared with 43 and 51 days respectively after crushing the sural nerve in adults at this level. It may be noted, however, that such a speedy recovery of sensory function in the sural area has been, as mentioned before, observed in 5 cases in which the sural has been cut and resected and in which there has been no possibility of regeneration, except by local extension of fibres from adjacent nerves through the skin plexuses.

In contrast to the speedy recovery in baby rabbits the recovery of sensation in very old animals has been found to be delayed. These old animals are characterized by an ingrowth of nails and a sleepy behaviour. Case 570 was of particular interest. The sensory recovery was very delayed and in addition no motor recovery had occurred at the calculated time on the side on which a suture was made at the level of the knee. Diagnosis of failure of the junction was made. At reoperation, however, the junction was found very satisfactory and electrical stimulation above the junction showed definite contraction of the muscles of the peroneal group. Five weeks after this exploration recovery in the autonomous zone did actually begin, but was very delayed and complete only after 171 days. The degree of sensory recovery was particularly poor in the distal parts of the autonomous zone.

3. Recovery and infection.-No special experiments have been undertaken to investigate the effect of infection on the process of recovery but it has been possible to make some occasional observations on animals accidentally infected which allowed us to assess the influence of infection on the process of recovery.

The infections found were small abscesses confined to fascia or skin stitches, or superficial suppurations of the skin which later granulated. The abscesses frequently were encapsulated containing pus and Staphylococcus aureus. Abscesses confined to stitches or even big abscesses infiltrating the spaces between the fascia had no effect on the final recovery as long as there was no great pressure or interruption of the nerve fibres by the abscess. Buo there was usually a delay in the beginning of the first stage of recovery, namely in the area of overlas with adjacent nerves. Moreover in two cases besides the effect on overlap there were also abnormalities in the recovery of sensation due to regeneration. These cases will be described in detail.

In animal 469 the peroneal nerve was crushed on both sides at the same level. On the right side the following process of recovery was observed. Two months after operation there was still a big area of analgesia which included the dorsum of the foot and the anterior surface of the shank up to the crista tibiæ. Then a rather quick shrinkage set in and in 3 months after the operation only the dorsum of the foot distally to a line just below the malleolar line was analgesic. In the next month there was only slight shrinkage. The first positive response was obtained $12 \mathrm{~mm}$. distal from the malleolar line and no further recovery took place. On the left side the same area of analgesia was found after the operation as on the right side. But after 54 days the first positive response was obtained in the malleolar line. A regular process of recovery in the autonomous zone followed, yet the recovery advanced exceptionally slowly. Total recovery was recorded after 132 days, a much longer time than was ever observed in other cases where the nerve was crushed at the same level. At biopsy a capsulated abscess $5 \times 3 \mathrm{~cm}$. in diameter, was found on the right side, partly infiltrating the peroneal nerve. Pinching the peroneal nerve in the shank did not elicit reflex responses. The first positive sensory reaction was obtained $60 \mathrm{~mm}$. distal to the crush. 
This case shows that on the infected side there was no recovery in the autonomous zone and even recovery in the zone of overlap was delayed. Moreover recovery was delayed on the uninfected side.

A different effect was observed in animal 546 in which the peroneal nerve was crushed on both sides at the same level. On both sides there was an analgesia of the foot and the anterior surface of the shank (Fig. 13, line 1). Recovery in the overlap

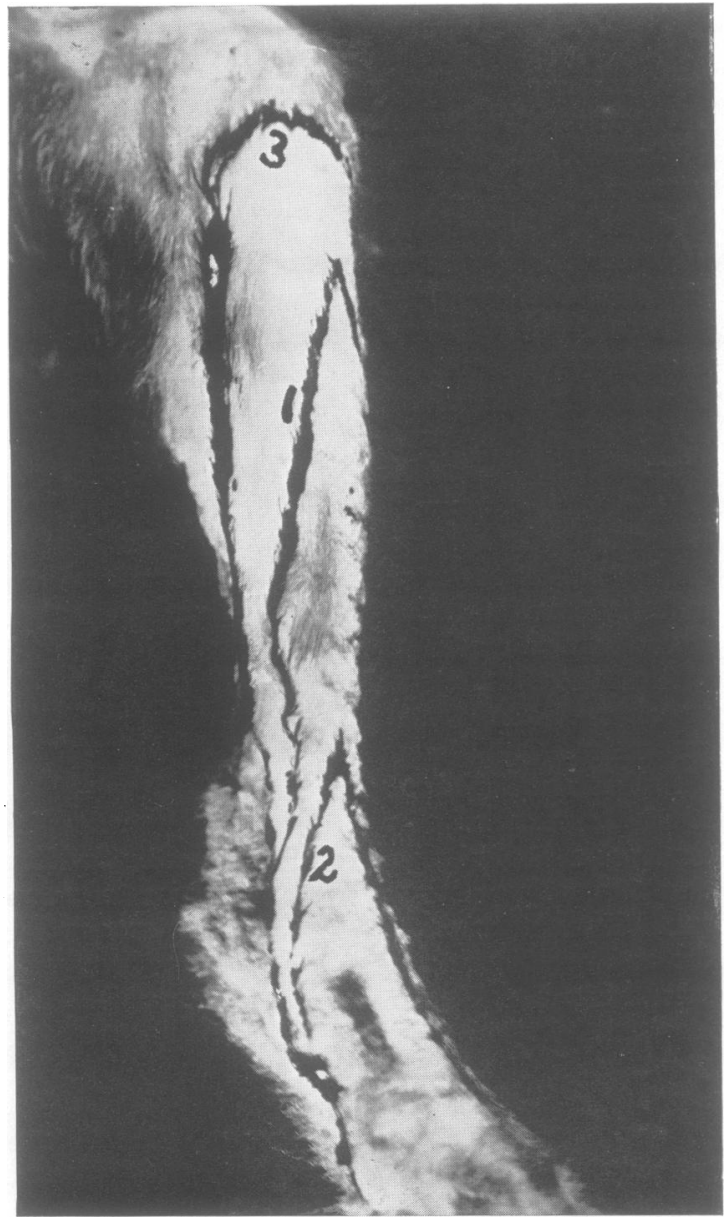

FIG. 13.-Shows the effect of infection on the recovery in overlap zones. Line 1 indicates the area of sensory loss 1 day after operation. Line 2, 5 days after operation. Line 3 the reversal of recovery after infection of the opposite leg.

zone set in very early and 5 days after the operation only the lower third of the shank was still analgesic (Fig. 13, line 2). Two weeks after the operation the animal became ill with a severe lymphangitis, edema of the shank and hydrocele. The skin was red and felt very hot, the animal was feverish and in bad condition. At the next examination 3 days later there was a complete analgesia of the dorsum of the foot and of the shank above the crista tibiæ on the uninfected side (Fig. 13, line 3). In the following days the edema diminished and the skin of the lateral part of the shank became necrotic. Very quick healing set in and new skin was formed which covered the defect. Recovery of sensation began again, proceeding in a regular way and being complete in 86 days on the formerly infected and 93 days on the uninfected side. Thus recovery in the autonomous zone proceeded even quicker on the infected side, being complete 1 week earlier than on the uninfected side.

Superficial infections (suppurating of the skin immediately after the operation) affect only the first stage of recovery, namely they delay recovery in overlap zones. This delay was found in 6 cases.

Rate of Advance of Sensory Recovery in the Skin

The rate of advance of "regeneration" in a nervecan be measured by a variety of methods, one of which is to record the advance of the margin of algesia over the skin (Gutmann et al., 1942). The examinations made during the present investigation have given ample opportunity to expand the observations already reported and the results of these are given in Tables I and II.

Table I.-The Rate of Recovery in the Autonomous Zone of the Peroneal Nerve after Crushing at Different Levels.

Length of auto-

Animal nomous Recovery Level of Rate Average

\begin{tabular}{|c|c|c|c|c|c|}
\hline 582 & $\begin{array}{l}60 \\
64 \\
74 \\
66 \\
55 \\
59 \\
56 \\
70 \\
60 \\
69 \\
48 \\
78 \\
58 \\
66 \\
73 \\
76 \\
80\end{array}$ & 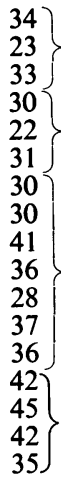 & $\begin{array}{l}\begin{array}{c}\text { Malleolar } \\
\text { line }\end{array} \\
\text { At knee }\end{array}$ & $\begin{array}{l}1.76 \\
2.78 \\
2.24 \\
2.20 \\
2.50 \\
1.90 \\
1.83 \\
2.33 \\
1.48 \\
1.92 \\
1.71 \\
2.10 \\
1.67 \\
1.57 \\
1.62 \\
1.80 \\
2.29\end{array}$ & $2 \cdot 26$ \\
\hline
\end{tabular}

Table II.-The Rate of Recovery in the AutonoMOUS Zone of THE Peroneal after Suturing IN THE MIDDle AND UPPER THIRD OF THE $ᄋ$ THIGH.

\begin{tabular}{|c|c|c|c|}
\hline & $\begin{array}{l}\text { Length of } \\
\text { autonomous }\end{array}$ & Recovery & $\mathrm{mm} / \mathrm{day}$ \\
\hline & zone & in days & mm./day \\
\hline 485L. & 30 & 60 & $\begin{array}{l}2.00 \\
1.49\end{array}$ \\
\hline $\begin{array}{l}486 \mathrm{~L} . \\
569\end{array}$ & $\begin{array}{l}37 \\
56\end{array}$ & 78 & 1.39 \\
\hline 589 & 47 & 66 & 1.40 \\
\hline 604 & 34 & 68 & $2 \cdot 00$ \\
\hline 670 & 56 & 62 & $1 \cdot 11$ \\
\hline & & erage rate & 1.57 \\
\hline
\end{tabular}

Since the margin of recovery does not always $\frac{0}{0} \frac{0}{0}$ advance smoothly it is clear that there are consider- $\stackrel{?}{\mathbb{P}}$ able difficulties in estimating the rate of regeneration $\stackrel{\odot}{\varnothing}$ by this method, these difficulties being of course 
more severe after suture when the recovery is more irregular, than after a crush injury. Nevertheless by taking the same precautions in estimating each case much of the uncertainty may be eliminated. The rate of regeneration has been estimated by calculating the time taken for the margin of analgesia to advance from a definite datum line, i.e. the first line beyond which all recovery could clearly and unambiguously be ascribed to regeneration of fibres of the damaged nerve in the autonomous zone, to the tips of the toes. The examinations have been made weekly, and thus the time of "complete recovery" may have been over-estimated in cases in which recovery has been found nearly complete at one examination and complete at the next. This error has been avoided by taking in these cases the time of complete recovery as the mean of the last two observations. The distance between these lines, divided by the number of days involved, then gives an estimate of the rate of advance of the process of recovery of algesia as recorded in this way.

The rates given in Tables 1 and 2 have been calculated in this way. By measuring the distance between the lines recorded at intermediate examinations we can make estimates of the rate of regeneration over shorter periods. Fig. 14 shows a com-

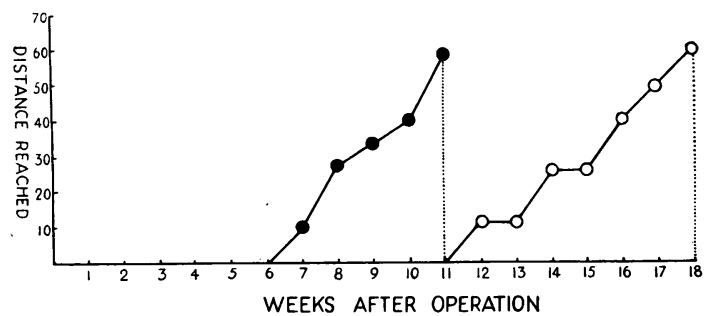

FIG. 14.-Diagram to show the onset and rate of recovery of sensation in the autonomous zone after crushing (line with black circles) and after suturing (line with white circles) of the peroneal nerve in the upper third of the thigh. The ordinates indicate the distance of the line of analgesia in $\mathrm{mm}$. from start of the autonomous zone, the abscissæ the weeks after operation. The dotted lines show the time at which recovery was found to be complete. Note slightly steeper slope of the line, earlier start of recovery in autonomous zone and quicker complete recovery after crushing the nerve.

parison of progress and rate of regeneration in the autonomous zone after crushing and after suturing the peroneal nerve. The figure shows graphically the later onset and slower progress of recovery in the autonomous zone after suturing the nerve. The late onset of recovery in the autonomous zone after suturing is of particular interest and indicates a longer waiting period ("peripheral delay") occupied, presumably by additon of fibres and a process of maturation. This period seems to be longer than after crushing. Calculating the time of arrival of the first axons in the beginning of the autonomous zone of the peroneal nerve below the malleolar line (Gutmann et al., 1942) these should arrive there 41 days after crushing and 53 days after suturing the nerve. From Fig. 14 it can be seen that recovery in the autonomous zone actually starts in the
6 th week after crushing but is declared till the 11 th week after suture.

The estimates show more irregular fluctuations than those obtained by the consideration of longer distances and times, and no doubt part of this fluctuation is due to errors of testing and random change in the state of the animal. Especially after sutures there are periods during which the margin of algesia changes little or not at all, and others in which it moves forward fast, almost by jumps.

Not enough animals have been examined to decide the question whether recovery of sensory function proceeds at a different rate after lesions of different nerves, but no very obvious signs of differences have appeared. Thus in 4 animals in which the tibial nerve was crushed about $200 \mathrm{~mm}$. from the end of the toes the average time of final recovery was 72 days compared with 78 days after peroneal lesions made at the corresponding level.

In attempting to summarize these figures we may consider (1) the average rate recorded from all cases in which the peroneal nerve was crushed, at whatever level: $1.98 \pm 0.09 \mathrm{~mm}$./day. (2) The average rate from all cases in which the peroneal nerve was severed and sutured: $1.57 \pm 0.15 \mathrm{~mm}$./day. In Table 1 the results are also classified according to the level of the lesion, and although the results from each level are rather few they show a tendency to lower rates from the more distant lesions. This is a most interesting result for the solution of the problem of the effect of distance on rate of res generation.

As has already been pointed out (Gutmann et al:, 1942) the question of whether the rate varies with the level of the injury cannot be settled by dividing time for recovery into distance from lesion to endorgan. If this were done the delay in the scar and in the end organ would enter in varying proportional extents into the calculation. In the present instance, however, where the rate is estimated between two points on the skin all other factors except distance can be regarded as constant. The results therefore suggest that regeneration in skin becomes slower as the distance between lesion and endorgan increases. This might be due to various factors, for instance, the increasing length of the nerve to be " maintained," the greater collapse of the Schwann tubes into which new fibres must grow (Holmes and Young, 1942), or the greater scatter in time of arrival of fibres in the skin. We shall see that the first two factors mentioned probably do not operate.

A further question is whether the rate of advance of regeneration in the nerve becomes slower towards the periphery. This can be examined by plotting the time necessary for complete recovery of algesia against distance of the lesion. This was the method previously adopted (Gutmann et al., 1942) to determine the rate of advance of functionally completed sensory fibres in the nerve. Further figures of the same sort are now available and given in Fig. 15. The most interesting feature in which these results add to those already published is that they include figures for the time of recovery after crushing the 
peroneal nerve at the malleolar line. We therefore have a set of data of the time necessary for recovery after lesions at a wider range of distances from the end organ (69 to $282 \mathrm{~mm}$.) than it is possible to obtain with any other technique in the rabbit. If the rate of regeneration is slower the longer the stretch of nerve which must recover we should expect that the points of Fig. 15 would not lie as a straight line. Unfortunately the data show too much scatter to enable this to be tested directly. However, inspection of the graph gives the impression that the rate of regeneration in the nerve does not fall off seriously as the distance to be repaired increases. If then we assume a linear relationship, the calculated rate of regeneration, including all animals in which the peroneal nerve has been crushed, is $2.48 \pm 0.26 \mathrm{~mm}$./day, which agrees

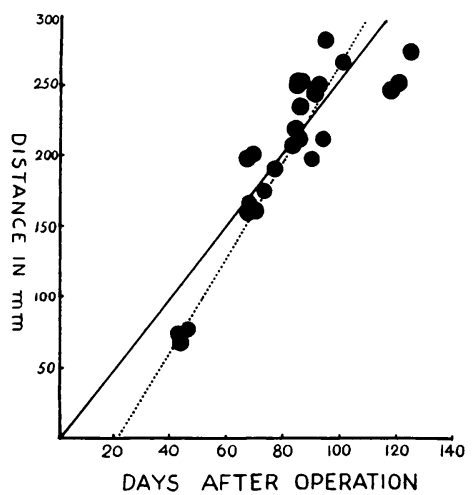

FIG. 15.-Graph showing the times of complete recovery plotted against level of the lesion after crushing the peroneal nerve at varying distances from the skin of the toes. The full line is the regression line, arithmetically calculated for all the points, the dotted line is fitted so as to pass through the low readings.

reasonably with that already given of $2 \cdot 74 \pm 0 \cdot 44$ $\mathrm{mm}$./day. However, it will be noticed that arithmetic calculation of the regression line has failed to give due weight to the three observations in which the nerve has been crushed very low. If we drew a line to pass nearer these points (which cannot be greatly in error) the rate would appear higher and the latent period longer. The dotted line shown in Fig. 15 is drawn in this way and gives a rate of regeneration of $3.35 \mathrm{~mm}$./day and a latent period of 22 days. These are probably better estimates than those arithmetically derived. This estimate agrees closely with the results from the paired data (Gutmann et al., 1942) obtained by crushing the peroneal nerve high on one and low on the other side of the same animal, which gave a rate of regeneration of $3.39 \mathrm{~mm}$./day.

Fig. 16 shows the results after severance and suturing of the peroneal nerve. The data include only one case in which the suture was done at the malleolar line and thus calculations of a regression line would be invalidated by an overweight of the cases in which sutures were performed higher up in the thigh. Further sutures were attempted at the malleolar line, but suturing a thin nerve at a point of mechanical stretch proved to be technically difficult and only the one satisfactory result has been achieved.

Including the datum from the suture at the malleolar line it appears to be difficult to fit a straight line, and the impression is gained that the rate falls off, being slower with the more distant lesions. As a compromise, therefore, the best possible line has been fitted to all points but so as to pass through the lowest point, which in any case cannot involve great error. This gives a rate of regeneration of $2.46 \mathrm{~mm}$./day which is slower than after crushing and gives a definitely longer latent period, 40 days.*

Sensory recoveries after suture show serious variations which seem to be characteristic of this procedure. In view of these variations it did not seem likely that it would be possible to obtain more

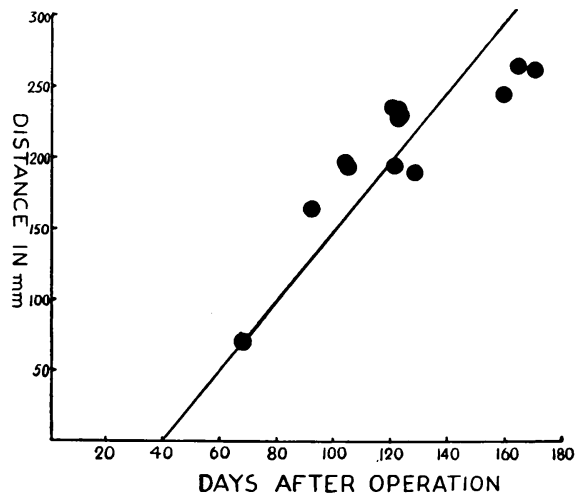

FIG. 16.-Graph showing the times of complete recovery plotted against level of the lesion after severance and suture the peroneal nerve at varying distances from the skin of the toes.

satisfactory data. Moreover, it must be remembered that calculation of the rate of regeneration made from these data rests on the assumption that it is constant over the whole stretch of the nerve. But, as pointed out, the data suggest a falling off of the line with the more distant lesions.

It can be concluded therefore that the rate of regeneration is slower after suturing than after crushing, that the latent period is longer after suturing, and that the rate of regeneration possibly falls off with the more distant lesions.

The data and those obtained from observations on recovery of motor function (Gutmann et al., 1942) allow a comparison of the rate of functional regeneration of motor and sensory fibres. The paired data obtained from observations on recovery of motor function give a rate of $3.10 \mathrm{~mm}$./day, those from observations on sensory recovery $3.39 \mathrm{~mm}$./day. A similar difference appears between the rates calculated from all data available, namely, $2.77 \mathrm{~mm}$./day for motor fibres (Gutmann, 1942) and $3.35 \mathrm{~mm}$./day for pain fibres. A slightly quicker recovery of pain fibres, these being the only ones

* A single observation only was available of an animal in which the peroneal nerve was sutured high on one and low on the other side. The rate of regeneration calculated from this experiment (Gutmann The rate of regeneration calculate
et al., 1942) was $2.0 \mathrm{~mm}$./day. 
we are considering in our sensory examinations, is to be expected as they are presumably unmedullated or thinly medullated fibres and their functional completion (Gutmann et al., 1942) will therefore need less time than that of the thicker and medullated motor fibres.

\section{Discussion}

Recovery of analgesic areas after nerve lesions in the rabbit may be said to take place by two processes. (1) By recovery due to compensatory action of the neighbouring nerves. (2) By recovery due to regeneration of the interrupted nerve in the autonomous zone.

\section{Recovery due to action of neighbouring nerves.}

(a) Recovery in overlap areas.-These areas naturally tend to occur chiefly where several nerves meet thus forming intermediate zones. According to the anatomical distribution of the overlapping nerves characteristic patterns of the intermediate zones in the sensory supply of individual peripheral nerves are found. Pure cutaneous nerves such as the sural, internal saphenous and lateral cutaneous nerve of the thigh show extensive overlap on both the proximal and distal borders, whereas in mixed nerves such as the tibial and peroneal the overlap in their distal distribution is far less or almost negligible. Accordingly the exact pattern of recovery due to overlap depends on this anatomical arrangement.

Although the intermediate zones contain intact fibres for the whole time after operation they do not immediately become responsive. In young animals their recovery may be complete within a few days. Thus the whole of the front of the leg of 2 rabbits, 1 month old, showed responses 3 days after interruption of the peroneal nerve. More usually, however, the fibres within these zones only become fully responsive after 2 to 4 weeks, the rule being that those in the areas nearest to the autonomous zone recover last. The question arises what process of readjustment occurs during the " recovery" of an intermediate zone. It might be in the skin itself, in the nerve trunk or in the C.N.S. That the peripheral factor is a very important one is shown by the fact that recovery of overlap zones is slower when the neighbouring nerves have been damaged by handling at the operation. On the other hand any factor which raises thresholds throughout the body (narcosis, infection, age, or general bad conditions) may also cause a reversal of recovery in an intermediate zone, even though it does not directly damage either the skin or the nerves. These overlap zones, therefore, when one of the nerves to them has been cut, contain too few fibres to function properly. It was particularly striking after peroneal lesions how long the large intermediate zone of this nerve over the antero-lateral aspect of the shank, remained hypoalgesic after recovery.

(b) Recovery by extension of fibres from adjacent nerves.-A further factor in the "recovery" due to action of adjacent nerves is the process of extension of fibres into the autonomous zone. There is little to add on this subject to what has already been said (Weddell et al., 1941). The process is naturally seen at its most marked where a small zone of loss. of sensation is surrounded by other nerves, e.g. in the sural nerve. We have confirmed that the autonomous zone of this nerve may become completely sensitive even in the absence of any possibility of growth down the nerve trunk. This was found particularly in young animals. But certainly the local extension cannot replace regeneration through the nerve in the recovery of larger areas. In mixed nerves such as the peroneal the extension of fibres from adjacent nerves into a denervated area is at most of subordinate importance for restoration of sensory function. Thus in 4 animals recovery through the peroneal nerve could not take place, and the margin of analgesia remained approximately at the malleolar line for 180 days.

\section{Recovery by regeneration of the nerve.}

Recovery within an analgesic autonomous zone due to true regeneration advances approximately from above downwards in all the examined nerves of the rabbit. The forward advance is most marked in the areas at the extremities of the limbs. In all the areas and especially those which are broad and lie higher up the limb, the margin of recovery advances faster at the edges than the centre of the area, so that towards the end recovery proceeds more or less "concentrically." This phenomenow is observed in the recovery of many areas in man? The exact pattern of recovery will depend on the anatomical arrangement of the branches within the area. Thus observations on recovery of sensation show that the visible pattern of recovery, consisting in centrifugal advance and concentric shrinkage is the same after lesions of all nerves.

The margin of algesia makes a general advance, however, which is sufficiently clear to allow estimates of its rate. As already pointed out (Gutmann et al., 1942), the rates which we have obtained are not very different from those which can be calculated from the results of other workers, e.g. Trotter and Davies (1909) on the human skin.

Careful use of the method of studying rate of advance of the algesic area on the dorsum of the foot of the rabbit gives a method for studying the effect of various factors on the process of sensory recovery. The nature of the lesion appears to be the most important of such factors. After crushing the nerve at a single point recovery advances on the foot quicker than after severance and suture. Moreover in the second case there are fewer points from which response can be elicited. These differences agree with those which have been found in all the studies on rate of regeneration (Gutmann et al., 1942) and one of us (Gutmann, 1942) has recently found exactly comparable differences in studies of motor recovery. It is not possible at present to be certain of the reason for the great success of nervous regeneration after a nerve has 
been crushed at a single point. The Schwann tubes maintain their continuity after such an operation (Young, personal communication), and it seems probable that the good regeneration is due largely to the large number of correct re-connections which it allows.

This is substantiated by analogous clinical observations made by one of us (L.G.), in cases of complete lesions in continuity of the musculospiral nerve due to alcohol or $\mathrm{CO}_{2}$ intoxication and others following fracture of the humerus in the middle of the upper arm, also recently in a case of a plexus lesion of inflammatory genesis (Guttmann and Pennybacker, 1942). All these cases showed the classical signs of a complete lesion but made an excellent and complete functional recovery. On the other hand there are lesions in continuity after crushing which only show imperfect functional recovery similar to that found after division of the nerve. These are cases in which considerable length of nerve has been crushed, probably producing rupture of a greater number of Schwann tubes and therefore giving less chance of correct re-connections. In this connection the cases described here of delayed and imperfect recovery in rabbits after extensive crushes are of interest.

The distance of the lesion from the skin plays a part in determining the speed of advance of recovery, the more distant lesions showing a slower advance of margin of recovery in the skin and perhaps also in the nerve trunk. This is probably to be interpreted as due to the scattered arrival of new fibres, though this cannot be decided for certain.

\section{Summary}

1. The maximal and autonomous zones of the cutaneous distribution of various peripheral nerves of the rabbit have been determined and the process of recovery of cutaneous sensation to nociceptive stimuli studied after interruption of these nerves.

2. Recovery of sensation in an analgesic area is a complex process comprising three main phases: (1) Recovery in zones of overlap by the progressive resumption of function by fibres of adjacent nerves. (2) Extension of these fibres into the denervated area. (3) Regeneration of the interrupted nerve.

3. Recovery in zones of overlap occurs generally 2 to 4 weeks after interruption of the nerve although in young animals it may occur within a few days after operation.
4. Recovery in zones of overlap is peculiarly susceptible to various factors such as local damage of the skin supplied by adjacent nerves, transient block of these nerves as a result of operation and all processes which tend to raise nervous thresholds such as infection, sores, narcosis, and age.

5. Recovery in the autonomous zone of a nerve due to regeneration of the interrupted nerve proceeds in general in a downward direction. Especially in large areas recovery often advances faster at the edges than in the centre, so that the analgesic area shrinks concentrically. The visible pattern of recovery, consisting in centrifugal advance and concentric shrinkage is the same after lesions of all nerves.

6. The nature of the lesion is of decisive importance for time and degree of recovery. After suturing recovery is slower, more irregular, and the degree of recovery is definitely less than after crushing.

7. Crushing the nerve over a stretch of $4 \mathrm{~cm}$. delays recovery by 2 weeks and is followed by a lower degree of functional recovery compared with recovery after a single crush.

8. Study of the time of recovery after lesions at different levels gives an estimate of the rate of progress of functional regeneration down the nerve of $3.35 \mathrm{~mm}$./day with a latent period of 22 days after crushing and $2.46 \mathrm{~mm}$./day with a latent period of 40 days after suturing. Great variabilities after suturing make this estimate less reliable than after crushing.

9. The average rate of advance of the margin of algesia in the autonomous zone on the dorsum of the foot in 17 animals after interruption of the peroneal nerve at various levels is $1.98 \pm 0.09$ $\mathrm{mm}$./day when the nerve has been crushed and $1.57 \pm 0.15 \mathrm{~mm}$./day in 6 cases when it has been cut and sutured.

10. The rate of advance of the margin of algesia is higher $(2.3 \mathrm{~mm}$./day) when the nerve is crushed at the ankle than when it is crushed in the thigh (1.8 mm./day).

The authors are very indebted to Mr. J. Z. Young for his help and advice throughout the whole course of this work and for revision of the paper, to Mr. P. B. Medawar for assistance in the statistical work, and to Mr. F. K. Sanders for helpful criticism.

The expenses of this investigation were defrayed by a grant from the Rockfeller Foundation to the Department of Zoology. The authors also wish gratefully to acknowledge a grant by the Nuffield Committee for the Advancement of Medicine.

\section{REFERENCES}

Gosset, A., and Bertrand J. (1938). J. chir., 51, 481.

Gutmann, E., Guttmann, L., Medawar, P. B. and Young, J. Z. (1942). J. exp. Biol., 19, 14.

Gutmann, E. (1942). J. Neurol. Psychiat (in press).

Guttmann, L., and Pennybacker, J. (1942). To be published.

Holmes, W., and Young, J. Z. (1942). J. Anat., Lond. 77, 33 .

Huber, G. C. (1919). Arch. Neurol. Psychiat., Chicago, $2,466$.

Muesch, H. J., and Roessel, W. (1941). Klin. Wschr., 23, 599 .
Tangari, C. (1940). Arch. Sci. méd., 65, 331.

Takimoto, G. (1916-1917). Mitt. med. Fak., Tokio, 16, 73.

Trotter, W., and Davies, H. M. (1909). J. Physiol., 38134.

Vanlair, C. (1882). Arch. Biol., Paris, 3, 379.

(1887). Ibid., 7, 433.

Weddell, G., Guttmann, L., and Gutmann, E. (1941). J. Neurol. Psychiat., 4, 206.

Young, J. Z., and Medawar, P. B. (1940). Lancet, $2,126$. 